\title{
Mechanism of miRNA-141-3p in Calcium Oxalate-Induced Renal Tubular Epithelial Cell Injury via NLRP3-Mediated Pyroptosis
}

\author{
Xiu-Guo Gan Zhi-Hao Wang Hai-Tao Xu \\ Department of Urology, First Affiliated Hospital of Harbin Medical University, Harbin, China
}

\section{Keywords}

Renal calculi · Renal tubular epithelial cells · Calcium oxalate $\cdot$ Pyroptosis $\cdot \mathrm{miR}-141-3 p$

\begin{abstract}
Background/Aims: Renal calculi represent a prevalent disorder associated with mineral deposition in renal calyces and the pelvis. Aberrant microRNA (miRNA) expression is implicated in renal injury. This study investigated the mechanism of miR-141-3p in calcium oxalate ( $\mathrm{CaOx})$ crystal-induced renal tubular epithelial cell (RTEC) injury. Methods: Human RTECs HK-2 cells were treated with $\mathrm{CaOx}$ crystals to induce RTEC injury. Cell viability was evaluated using Cell Counting Kit-8 assay, and apoptosis was measured using flow cytometry. The contents of lactate dehydrogenase (LDH), malondialdehyde (MDA), superoxide dismutase (SOD), interleukin (IL)- $1 \beta$, and IL-18 were measured using enzyme-linked immunosorbent assay kits. The expressions of NLRP3, cleaved caspase-1, and GSDMD-N were detected using Western blot. miR-141-3p and NLRP3 expressions were determined using reverse transcription quantitative polymerase chain reaction. The binding of miR-141-3p and NLRP3 was validated using a dual-luciferase assay. The role of NLRP3 in the protection of miR-141-3p on RTEC injury was verified using func-
\end{abstract}

Karger@karger.com www.karger.com/kbr

Karger"

BOPEN ACCESS
(C) 2022 The Author(s)

Published by S. Karger AG, Basel

This is an Open Access article licensed under the Creative Commons Attribution-NonCommercial-4.0 International License (CC BY-NC) (http://www.karger.com/Services/OpenAccessLicense), applicable to the online version of the article only. Usage and distribution for commercial purposes requires written permission. tional rescue experiments. Results: $\mathrm{CaOx}$ crystals induced RTEC injury, manifested as attenuated cell viability, enhanced apoptosis, elevated intracellular LDH and MDA levels, and decreased SOD level. Pyroptosis of RTECs was enhanced by $\mathrm{CaOx}$ crystal induction, evidenced by elevated expressions of cleaved caspase-1, GSDMD-N, IL-1 $\beta$, and IL18. miR-141-3p expression was reduced in $\mathrm{CaOx}$ crystal-induced RTECs. miR-141-3p overexpression alleviated $\mathrm{CaOx}$ crystal-induced RTEC injury and suppressed pyroptosis of RTECs. miR-141-3p bound to NLRP3 and thereby repressed NLRP3 expression. NLRP3 overexpression reversed the protective effect of miR-141-3p overexpression on RTECs. Conclusion: miR-141-3p repressed NLRP3-mediated pyroptosis by suppressing NLRP3 expression, thus protecting $\mathrm{CaOx}$ crystal-induced RTEC injury.

(c) 2022 The Author(s).

Published by S. Karger AG, Basel

\section{Introduction}

Renal calculi are mineral depositions in renal calyces and pelvis that are free or attached to renal papillae [1]. Although many renal calculi are asymptomatic for a long period, the problems of pain, infection, and obstruction are increasing, and over $50 \%$ of patients have recurrent
Correspondence to:

Xiu-Guo Gan, ganxiuguo0707@163.com 
renal calculi after 5 years [2]. Despite the considerable advances in the surgical treatment of removing renal calculi, the progress in preventing renal calculi recurrence is limited [3]. The high recurrence rate and a heavy surgical burden have increased the risks of renal function reduction and chronic renal injury in these patients [4]. Thus, exploring potent therapeutic targets to mitigate renal injury in patients with renal calculi remains an urgent issue.

Approximately $80 \%$ of renal calculi are initiated by the deposition of calcium oxalate $(\mathrm{CaOx})$ crystals [5]. $\mathrm{CaOx}$ stone matrix contains numerous protein molecules, which can facilitate the aggregation, nucleation, and growth of $\mathrm{CaOx}$ crystals within tubular cells and finally result in the formation of renal calculi [6]. The critical mechanism of renal calculi formation is the adhesion of $\mathrm{CaOx}$ crystals to renal tubular epithelial cells (RTECs) [7]. The interaction between $\mathrm{CaOx}$ crystals and RTECs stimulates the generation of reactive oxygen species, secretes massive inflammatory cytokines, and activates an inflammatory cascade in RTECs, leading to intrarenal inflammation and RTEC injury [8]. It has been demonstrated that $\mathrm{CaOx}$ crystals-triggered renal inflammation is mediated by the Nod-like receptor protein 3 (NLRP3) inflammasome [9]. NLRP3 inflammasome is a well-studied cytoplasmic protein complex that can activate caspase-1, which causes the maturation of interleukin (IL)- $1 \beta$ and IL-18 and then induces a proinflammatory form of cell death, known as pyroptosis [10]. NLRP3 inflammasomemediated pyroptosis in RTECs contributes to the progression of diverse kidney diseases [11]. Nevertheless, the exact effects and molecular mechanism of NLRP3-mediated pyroptosis in RTECs in renal calculi have not been elucidated before.

microRNAs (miRNAs) are a class of small endogenous noncoding RNAs (18-25 nt in length) that modulate gene expression at the posttranscriptional level [12]. Microarray analysis has identified several miRNAs with differential expression profiles in $\mathrm{CaOx}$ crystal-induced RTECs [13]. miR-141-3p has been reported to support the epithelioid morphology of RTECs and repress renal interstitial fibrosis [14]. However, the role of miR-141-3p in $\mathrm{CaOx}$ crystal-induced RTECs remains unknown. Intriguingly, miR-141-3p overexpression can also suppress the expressions of pyroptosis-associated proteins in high glucose-induced cardiomyocytes [15]. Hence, this study sought to determine whether miR-141-3p can play a role in CaOx crystal-induced RTECs by mediating NLRP3mediated pyroptosis, hoping to confer novel insights for the treatment of renal calculi with miRNA as a molecular target.

miR-141-3p Reduces CaOx-Induced RTEC Injury

\section{Materials and Methods}

\section{Cell Culture and Treatment}

Human RTECs HK-2 were purchased from American Type Culture Collection (Manassas, VA, USA) and cultured in Dulbecco's modified Eagle's medium/F12 containing 10\% fetal bovine serum (Gibco, Grand Island, NY, USA) and 1\% penicillin-streptomycin (Gibco, $100 \mathrm{IU} / \mathrm{mL}$ penicillin and $100 \mu \mathrm{g} / \mathrm{mL}$ streptomycin) at $37^{\circ} \mathrm{C}$ with $5 \% \mathrm{CO}_{2}$.

Cell injury was induced by $\mathrm{CaOx}$ crystals as described in the previous literature $[16,17]$ : the cells were cultured in the complete medium containing $0,0.1,1,2$, and $4 \mathrm{~mm} \mathrm{CaOx}$ crystals. After 24 $\mathrm{h}$, the cells and supernatants were collected for further analysis.

\section{Cell Transfection}

The expression of miR-141-3p or NLRP3 in cells was upregulated by miR-141-3p mimic (miR-mimic) or oe-NLRP3. miRmimic, oe-NLRP3, and their corresponding negative controls (miR-NC and oe-NC) were provided by GenePharma (Shanghai, China) and transiently transfected into HK-2 cells using Lipofectamine 2000 (Invitrogen, Carlsbad, CA, USA) in line with the manufacturer's instructions. The cells were collected $48 \mathrm{~h}$ after transfection for further analyses.

\section{Cell Counting Kit-8 Assay}

Cell viability was evaluated using the Cell Counting Kit- 8 assay kit (KeyGEN, Nanjing, China). Briefly, HK-2 cells were seeded into 6-well plates, and each well was added with $10 \mu \mathrm{L}$ Cell Counting Kit- 8 solution, followed by 2 -h incubation at $37^{\circ} \mathrm{C}$. The optical density at $450 \mathrm{~nm}$ was measured. The experiment was repeated 3 times independently.

\section{Flow Cytometry}

The apoptosis level was evaluated using flow cytometry. The $\mathrm{CaOx}$-treated and transfected HK-2 cells were rinsed with phosphate-buffered saline, suspended in annexin V-FITC binding buffer (Beyotime, Shanghai, China), and incubated with annexin VFITC (Beyotime) and propidium iodide (Beyotime). Finally, the apoptosis was analyzed using a flow cytometer (Beckman Coulter, Inc., Chaska, MN, USA).

\section{Reverse-Transcription Quantitative Polymerase Chain}

\section{Reaction}

The total RNA was extracted from HK-2 cells using TRIzol reagent (Invitrogen) and reverse transcribed into cDNA using the reverse transcription kit ( $\&$ \& Systems, Minneapolis, MN, USA). miR-141-3p was detected using the Taqman Advanced microRNA Assay (Thermo Fisher Scientific Inc., Waltham, MA, USA). mRNA was detected using the Universal SYBR Green PCR kit (Takara, Kyoto, Japan). PCR was performed using the 7500 Real-time PCR system (Applied Biosystems, Foster City, CA, USA), with U6 as the internal reference of miR-141-3p and GAPDH as the internal reference of NLRP3. The relative expression of genes was calculated using the $2^{-\Delta \Delta \mathrm{Ct}}$ method. The experiment was repeated 3 times independently. Table 1 shows the primer sequences.

\section{Western Blot}

The total protein was isolated from cells using the PROPREPTM protein extract (iNtRON Biotechnology, Seongnam, South Korea), and the protein concentration was determined us- 
Forward primer $\left(5^{\prime}-3^{\prime}\right)$

CCTCGTCTTGAGCTGAGAGC GCAAGCACCCGCTGCAAGC GATGCTGGCGCTGAGTACG CTCGCTTCGGCAGCACATA
Reverse primer $\left(5^{\prime}-3^{\prime}\right)$

AGGGCTCCCTGAAGGTTACT CTACCAAGAAGGCTCAAAGA GCTAAGCAGTTGGTGGTGC AACGATTCACGAATTGCGT

$\mathrm{RT}-\mathrm{qPCR}$, reverse transcription quantitative polymerase chain reaction; miR-141-3p, microRNA-141-3p; NLRP3, Nod-like receptor protein 3; GAPDH, glyceraldehyde-3-phosphate dehydrogenase.

ing the bicinchoninic acid kit (R\&D SYSTEMS). The protein samples were separated by SDS-PAGE, then transferred onto the polyvinylidene difluoride membranes, and blocked with $5 \%$ skim milk (BD Biosciences, Sparks, MD, USA) for $2 \mathrm{~h}$. Afterward, the diluted primary antirabbit monoclonal antibody cleaved caspase-1 (4199, 1:1000, CST, Boston, USA), rabbit monoclonal antibody GSDMD-N (ab215203, 1:1000, Abcam, Cambridge, UK), and rabbit polyclonal antibody NLRP3 (ab214185, 1: 1000, Abcam) were added for incubation overnight. Next, the secondary antibody goat anti-rabbit IgG H\&L (HRP) (ab6721, 1:2000, Abcam) was supplemented for 1-h incubation. Finally, the membranes were developed using enhanced chemiluminescence solution (EMD Millipore, Billerica, MA, USA). The intensity of each band in Western blot images was brightened using Image Pro Plus 6.0 (Media Cybernetics, San Diego, CA, USA). $\beta$-Actin was used as the internal reference. The experiment was repeated 3 times independently.

\section{Enzyme-Linked Immunosorbent Assay}

The levels of lactate dehydrogenase (LDH), malondialdehyde (MDA), superoxide dismutase (SOD), IL-1 $\beta$, and IL-18 were measured using enzyme-linked immunosorbent assay kits (Proteintech, Chicago, IL, USA).

\section{Bioinformatics}

The binding site of miR-141-3p and NLRP3 was predicted through the TargetScan database (http://www.targetscan.org/ vert_72/) [18].

\section{Dual-Luciferase Assay}

The synthesized NLRP3 gene fragment containing miR-141-3p binding site (NLRP3-WT) and NLRP3-MUT mutated according to the binding site was constructed into pmirGLO-reporter plasmids (Huayueyang Biotechnology, Beijing, China). The constructed plasmids were cotransfected with mimic-NC or miR-141-3pmimic into HK-2 cells. After 48 h, cells were collected and lysed. Luciferase activity was measured using the Firefly Luciferase Assay kit (K801-200; Biovision, Mountain View, CA, USA). All plasmids were supplied by GenePharma (Shanghai, China). The experiment was repeated 3 times independently.

\section{Statistical Analysis}

Data analysis and map plotting were introduced using the SPSS 21.0 (IBM Corp., Armonk, NY, USA) and GraphPad Prism 8.0 (GraphPad Software Inc., San Diego, CA, USA). The measurement data are expressed as mean \pm standard deviation. Compari- sons among multiple groups were performed using one-way or two-way analysis of variance (ANOVA), following Tukey's multiple comparison test. A value of $p<0.05$ meant a significant difference.

\section{Results}

CaOx Crystals -Induced RTEC Injury and miR-141$3 p$ Were Poorly Expressed in CaOx-Induced RTEC Injury

In this study, we established an RTEC injury model by treating $\mathrm{HK}-2$ cells with different concentrations of $\mathrm{CaOx}$ crystals $(0.1,1,2$, and $4 \mathrm{~mm})$ and found that $\mathrm{CaOx}$ crystals elevated the apoptosis level of HK-2 cells ( $p<0.05$; Fig. 1a) and reduced the cell viability ( $p<0.05$; Fig. $1 \mathrm{~b})$. The alternations of LDH, MDA, and SOD in HK-2 cells were also measured, and our results demonstrated that $\mathrm{CaOx}$ crystals notably elevated the contents of $\mathrm{LDH}$ and MDA, but reduced the content of SOD ( $p<0.05$; Fig. 1c-e). To determine whether miR-141-3p was implicated in the regulation of renal tubular epithelial injury, we detected miR141-3p expression in cells and found that miR-141-3p expression was reduced significantly after $\mathrm{CaOx}$ crystal treatment ( $p<0.05$, Fig. 1f). Briefly, CaOx crystals induced the injury of RTECs in a concentration-dependent manner and downregulated miR-141-3p expression in cells. According to our results, $\mathrm{CaOx}$ crystals at a concentration of $2 \mathrm{mM}$ were selected for the subsequent experiments.

\section{miR-141-3p Overexpression Alleviated CaOx Crystal-}

Induced RTEC Injury

To explore the regulatory effect of miR-141-3p on RTEC injury induced by CaOx crystals $(2 \mathrm{mM})$, we overexpressed miR-141-3p in HK-2 cells ( $p<0.05$, Fig. 2a). Overexpression of miR-141-3p significantly reduced the apoptosis level $(p<0.05$, Fig. $2 b)$ and enhanced cell viability $(p<0.05$, Fig. $2 c)$. After overexpression of miR-141- 


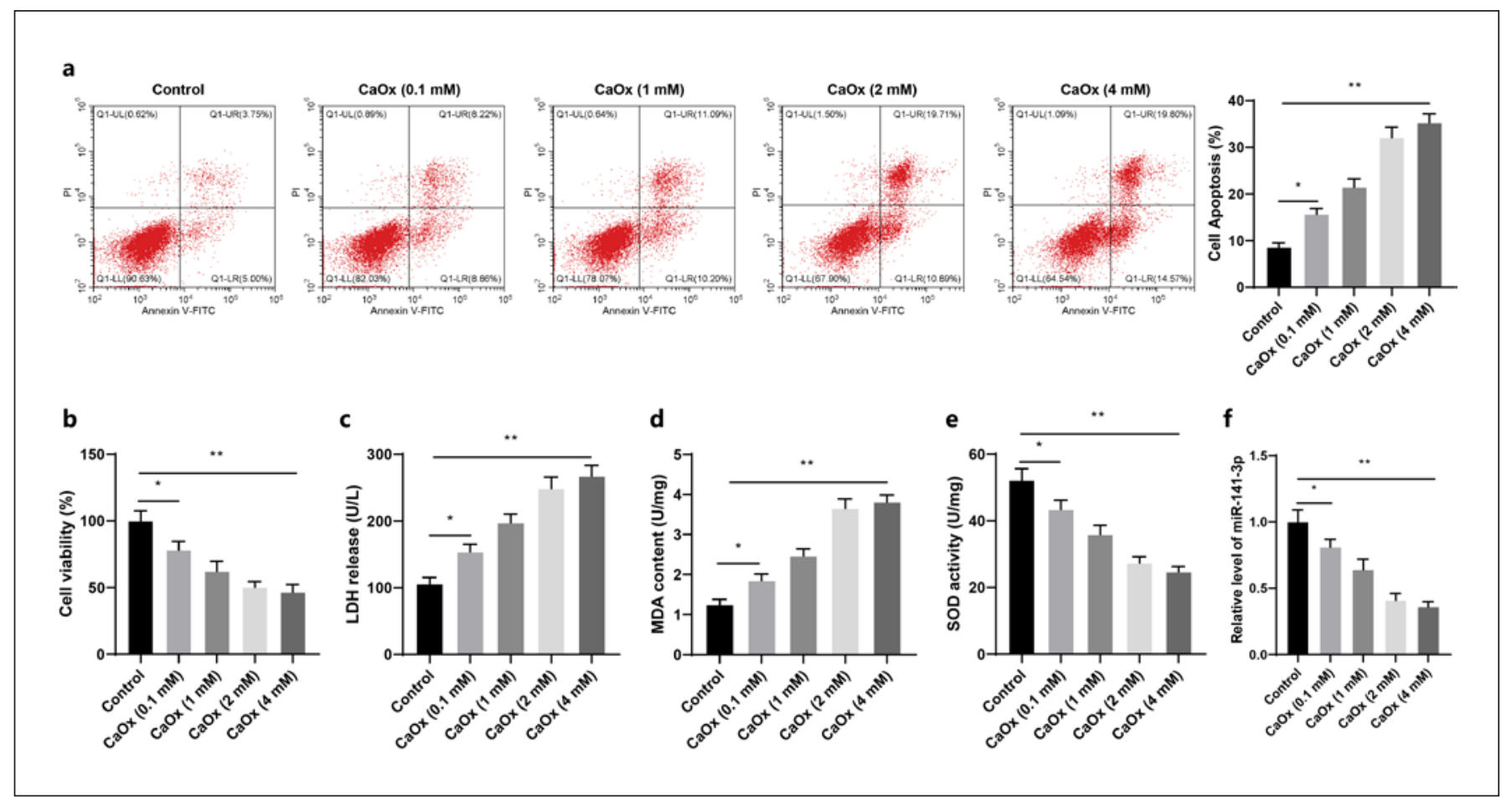

Fig. 1. CaOx-induced RTEC injury and miR-141-3p were poorly expressed in CaOx-induced RTEC injury. Different concentrations of $\mathrm{CaOx}$ crystals $(0.1,1,2$, and $4 \mathrm{mM})$ were used to induce RTEC injury. a Apoptosis level was measured using flow cytometry. b Cell viability was evaluated using the CCK-8 assay. c-e The contents of LDH, MDA, and SOD were detected using ELISA. $\mathbf{f}$ miR-141-3p expression was determined using RT-qPCR. The cell experiment was repeated 3 times independently. Data are presented as mean \pm standard deviation and analyzed using one-way ANOVA, followed by Tukey's multiple comparisons test, ${ }^{*} p<0.05$, ${ }^{* *} p<0.01$. RT-qPCR, reverse transcription quantitative polymerase chain reaction; ELISA, enzyme-linked immunosorbent assay; CCK-8, Cell Counting Kit-8.
$3 p$, the contents of LDH and MDA in cells were decreased $(p<0.05$, Fig. $2 \mathrm{~d}, \mathrm{e})$ and the content of SOD was increased $(p<0.05$, Fig. 2f). In short, overexpression of miR-141-3p reduced RTEC injury induced by $\mathrm{CaOx}$ crystals and improved cell viability.

miR-141-3p Overexpression Suppressed CaOx CrystalInduced Pyroptosis of RTECs

$\mathrm{CaOx}$ crystals can trigger inflammasomes to activate pyroptosis, resulting in epithelial cell injury [19]. Therefore, we speculated whether miR-141-3p alleviated $\mathrm{CaOx}$ crystal-induced RTEC injury by inhibiting the pyroptosis of RTECs. To test this conjecture, we first determined the NLRP3 protein level in HK-2 cells and found that the NLRP3 protein level was decreased notably after overexpression of miR-141-3p (Fig. 3a). The protein levels of cleaved caspase- 1 and GSDMD-N were decreased with the upregulation of miR-141-3p ( $p<0.05$, Fig. 3a). After overexpression of miR-141-3p, the levels of IL- $1 \beta$ and IL-
18 were also reduced notably ( $p<0.05$, Fig. $3 b$ ). All these indicated that miR-141-3p overexpression suppressed $\mathrm{CaOx}$ crystal-induced pyroptosis of RTECs.

\section{miR-141-3p Repressed NLRP3 Expression}

Inhibiting the activation of the NLRP3 inflammasome pathway reduces the release of inflammatory mediators, thereby improving $\mathrm{CaOx}$ crystal-induced renal tissue injury [20]. Through the TargetScan database (http://www. targetscan.org/vert_72/) prediction, it was found that there was a binding site between miR-141-3p and NLRP3 (Fig. 4a). The dual-luciferase assay validated the binding of miR-141-3p and NLRP3 ( $p<0.05$, Fig. $4 b$ ). Then, NLRP3 expression in RTECs was detected, and the results exhibited that $\mathrm{CaOx}$ crystals significantly elevated NLRP3 expression, while NLRP3 expression was notably declined after overexpression of miR-141-3p ( $p<0.05$, Fig. 4c). Briefly, miR-141-3p repressed NLRP3 expression by binding to NLRP3 in CaOx crystal-induced RTECs. 


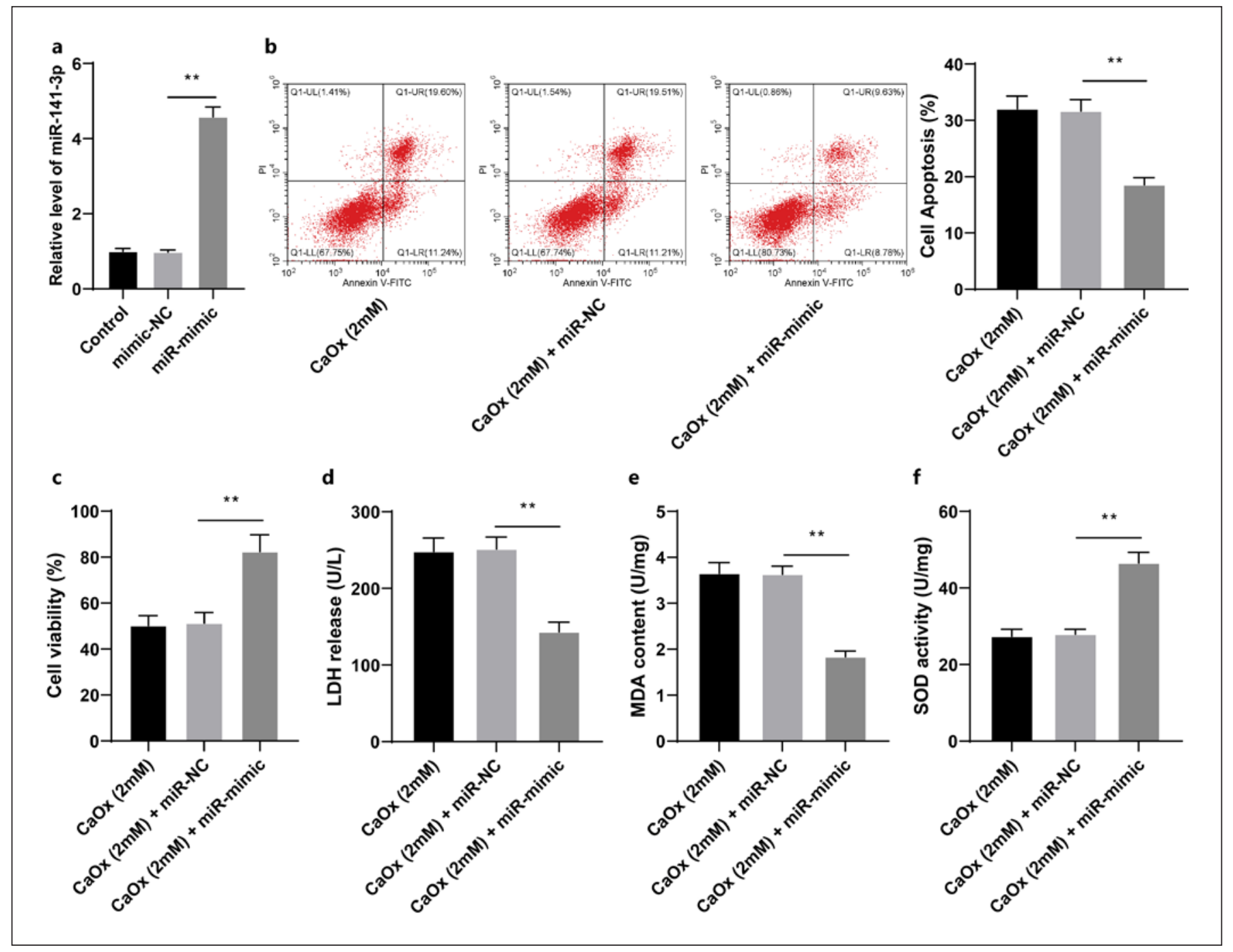

Fig. 2. miR-141-3p overexpression alleviated $\mathrm{CaOx}$ crystal-induced RTEC injury. RTEC injury was induced by $\mathrm{CaOx}$ crystals with a concentration of $2 \mathrm{mM}$. miR-mimic was transfected into the $\mathrm{CaOx}$ crystal-induced cells to upregulated miR-141-3p expression, with mimic-NC as the control. a miR-141-3p transfection efficiency was verified using RT-qPCR. b Apoptosis level was measured using flow cytometry. c Cell viability was evaluated using the CCK-
8 assay. d-f The contents of LDH, MDA, and SOD were detected using ELISA. The cell experiment was repeated 3 times independently. Data are presented as mean \pm standard deviation and analyzed using one-way ANOVA, followed by Tukey's multiple comparisons test, ${ }^{* *} p<0.01$. RT-qPCR, reverse transcription quantitative polymerase chain reaction; ELISA, enzyme-linked immunosorbent assay; CCK-8, Cell Counting Kit-8.

\section{NLRP3 Overexpression Reversed the Protective Effect} of miR-141-3p Overexpression on RTECs

To further verify that NLRP3 was involved in the protective effect of miR-141-3p on RTECs, we overexpressed NLRP3 in cells $(p<0.05$, Fig. 5a) and then conducted a combined experiment with miR-141-3p overexpression. Overexpression of NLRP3 significantly enhanced the apoptosis level and reduced cell activity $(p<0.05$, Fig. 5b, c), elevated the contents of $\mathrm{LDH}$ and MDA, and decreased the content of SOD ( $p<0.05$, Fig. $5 d$ ). In addition, after the combined treatment of NLRP3 overexpression and miR-141-3p overexpression, the protein levels of cleaved caspase- 1 and GSDMD-N in cells were increased notably $(p<0.05$, Fig. 5e), and the inflammation-related factors IL-1 $\beta$ and IL-18 were also elevated ( $p<0.05$, Fig. 5f). Briefly, NLRP 3 overexpression reversed the protective effect of miR-141-3p overexpression on RTECs. 


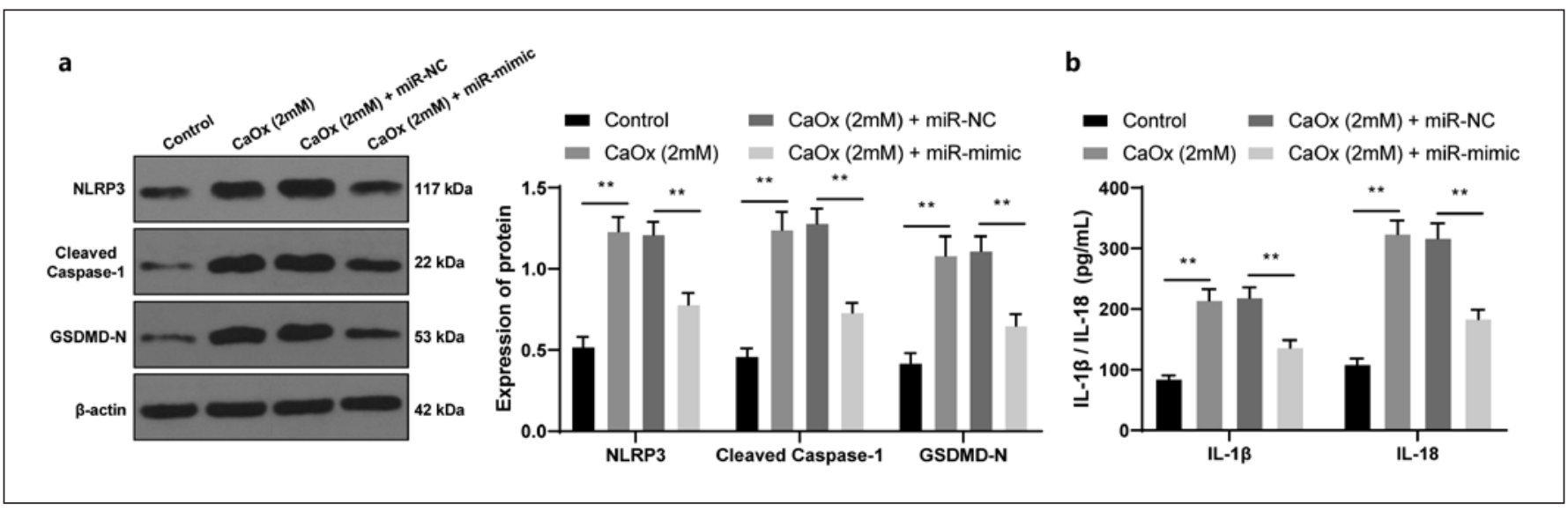

Fig. 3. miR-141-3p overexpression suppressed $\mathrm{CaOx}$ crystal-induced pyroptosis of RTECs. RTEC injury was induced by $\mathrm{CaOx}$ crystals with a concentration of $2 \mathrm{mM}$. miR-mimic was transfected into the $\mathrm{CaOx}$ crystal-induced cells to upregulated miR-141-3p expression, with mimic-NC as the control. a The protein levels of NLRP3, cleaved caspase-1, and GSDMD-N were detected using
Western blot. $\mathbf{b}$ The levels of IL-1 $\beta$ and IL-18 were detected using ELISA. The cell experiment was repeated 3 times independently. Data are presented as mean \pm standard deviation and analyzed using two-way ANOVA, followed by Tukey's multiple comparisons test, ${ }^{* *} p<0.01$. ELISA, enzyme-linked immunosorbent assay.

\section{a \\ NLRP3 WT}

miR-141-3p

5'-GGGGCUGCCAGACGCCAGUGUUC-3'

NLRP3 MUT 3'-GguAGAAAUGGUCUGUCACAAU-5' $:: \vdots: \vdots 1:$ 5'-GGGGCUGCCAGACGCGUCACUAC-3'

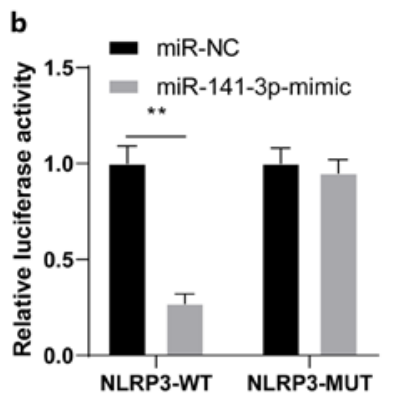

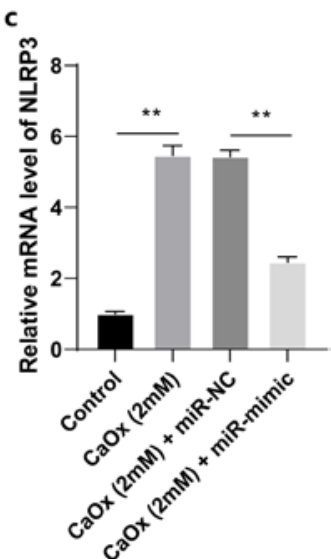

Fig. 4. miR-141-3p repressed NLRP3 expression. a The binding site between miR-141-3p and NLRP3 was predicted through the TargetScan database (http://www.targetscan.org/vert_72/). b The binding of miR-141-3p and NLRP3 was verified using a dual-luciferase assay. c NLRP3 expression was determined using RT-qPCR. The cell experiment was repeated 3 times independently. Data are presented as mean \pm standard deviation. Data in panel B were analyzed using two-way ANOVA, and data in panel (c) were analyzed using one-way ANOVA, followed by Tukey's multiple comparisons test, ${ }^{* *} p<0.01$. RT-qPCR, reverse transcription quantitative polymerase chain reaction.

miR-141-3p Reduces CaOx-Induced RTEC Injury

\section{Discussion}

Renal calculi are prevalent in all population groups with a rising incidence rate and often become lifelong trouble [2]. Abnormal aggregation of $\mathrm{CaOx}$ crystals in the kidney is one of the major causes of renal calculi $[16,17]$. Recently, an analysis of miRNA expression profiles in CaOx-induced renal calculi suggests the implication of miRNAs in the development of renal calculi [12]. It has been reported that miR-141-3p expression is reduced in RTEC injury [14]. This study elucidated that miR-141-3p overexpression alleviated $\mathrm{CaOx}$ crystal-induced RTEC injury by targeting NLRP3-mediated pyroptosis.

Renal calculi are predominantly composed of $\mathrm{CaOx}$ and exist in the form of monohydrate or dihydrate [1]. In the current study, we established a cell model of RTEC injury by treating HK-2 cells with different concentrations of $\mathrm{CaOx}$ crystals. The exposure of RTECs to $\mathrm{CaOx}$ can lead to a signaling cascade that causes apoptosis via the p38 mitogen-activated protein kinase pathway [21]. We found that $\mathrm{CaOx}$ crystal treatment notably enhanced the apoptosis of RTECs and reduced cell viability. CaOx-induced oxidative stress can result in RTEC injury and renal calculi formation [22]. We determined the contents of oxidative stress-related indicators including LDH, MDA, and SOD in $\mathrm{CaOx}$ crystal-induced HK-2 cells and found that $\mathrm{CaOx}$ crystals elevated the contents of $\mathrm{LDH}$ and MDA but reduced the content of SOD. Briefly, these results indicated that $\mathrm{CaOx}$ crystals induced RTEC injury. 


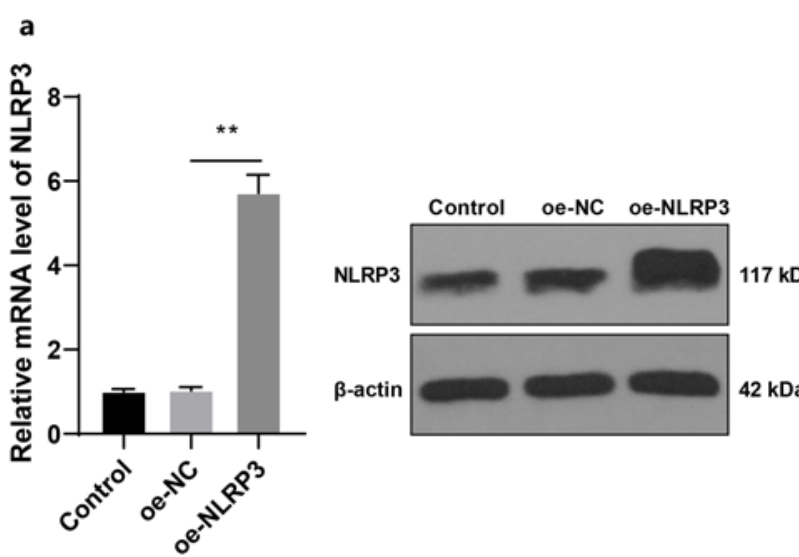

C
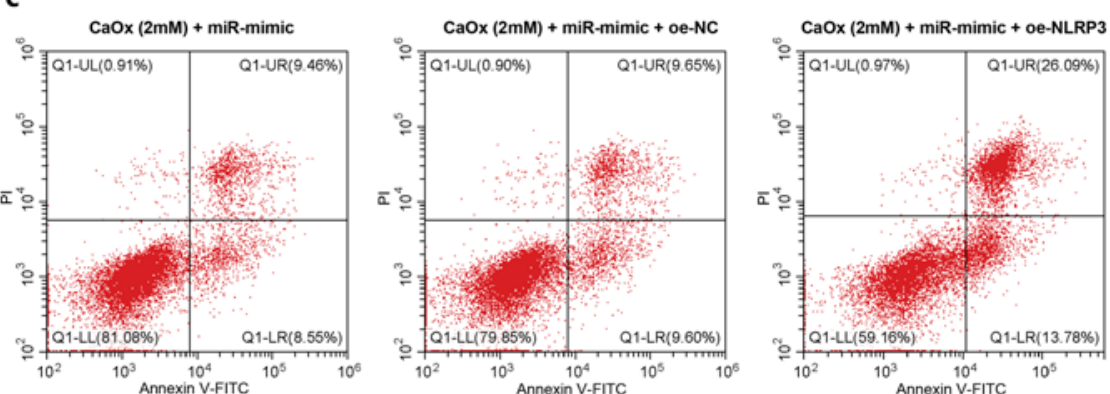

d

- $\mathrm{CaOx}(2 \mathrm{mM})+$ miR-mimic

- $\mathrm{CaO} \times(2 \mathrm{mM})+$ miR-mimic + oe-NC

- $\mathrm{CaOx}(2 \mathrm{mM})+$ miR-mimic

- $\mathrm{CaO} \times(2 \mathrm{mM})+$ miR-mimic + oe-NC

- $\mathrm{CaO} \times(2 \mathrm{mM})+$ miR-mimic + oe-NLRP3

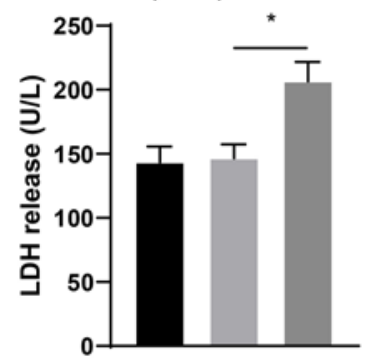

e

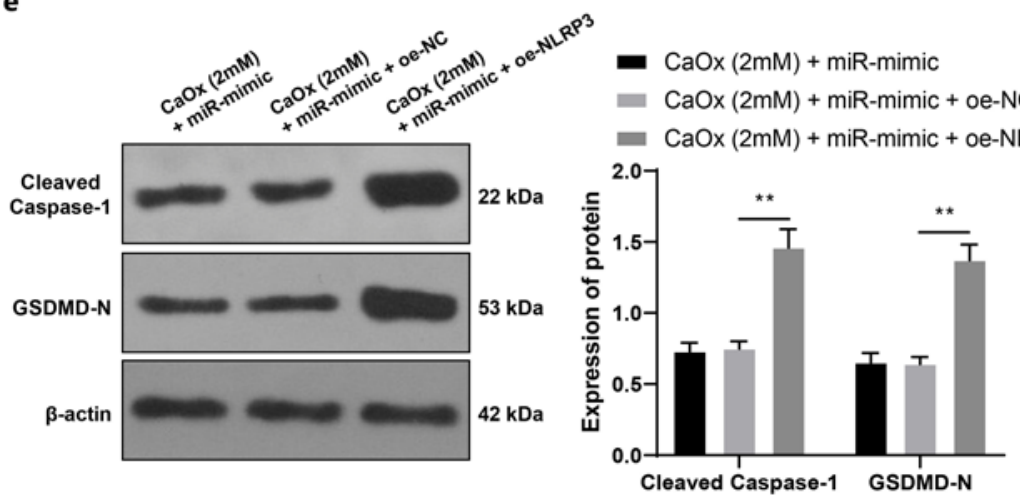

b

- $\mathrm{CaOx}(2 \mathrm{mM})+$ miR-mimic

= $\mathrm{CaO} \times(2 \mathrm{mM})+$ miR-mimic + oe-NC

- $\mathrm{CaO} \times(2 \mathrm{mM})+$ miR-mimic + oe-NLRP3

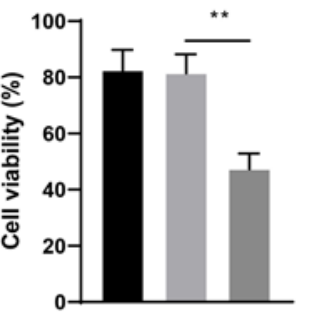

- $\mathrm{CaOx}(2 \mathrm{mM})+$ miR-mimic

- $\mathrm{CaOx}(2 \mathrm{mM})+$ miR-mimic + oe-NC

- $\mathrm{CaO} \times(2 \mathrm{mM})+$ miR-mimic + oe-NLRP3

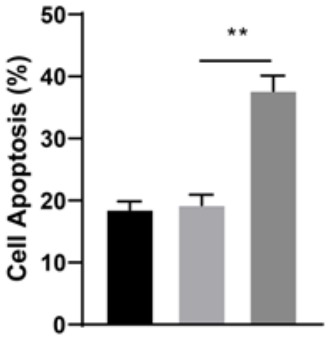

- $\mathrm{CaOx}(2 \mathrm{mM})+$ miR-mimic

- $\mathrm{CaO} \times(2 \mathrm{mM})+$ miR-mimic + oe-NC

- $\mathrm{CaO} \times(2 \mathrm{mM})+$ miR-mimic + oe-NLRP3

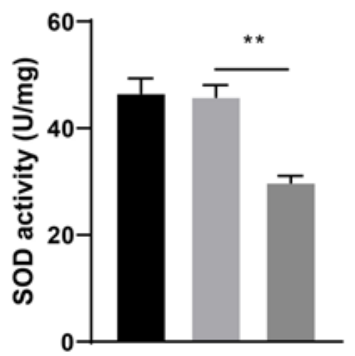

f

- $\mathrm{CaOx}(2 \mathrm{mM})+$ miR-mimic

- $\mathrm{CaO} \times(2 \mathrm{mM})+$ miR-mimic + oe-NC

- CaOx $(2 \mathrm{mM})+$ miR-mimic + oe-NLRP3

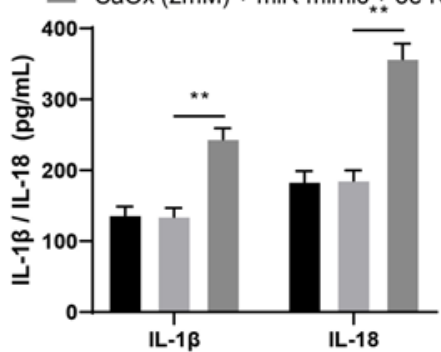

(For legend see next page.) 
miRNAs are a class of posttranscriptional gene modulators, which are implicated in the alteration of gene expression in renal cells induced by $\mathrm{CaOx}$ crystals [13] miR-141-3p expression is downregulated in the HK-2 cell model of renal fibrosis [14]. Nevertheless, the role of miR141-3p in CaOx crystal-induced RTEC injury remained unknown. We were the first to demonstrate that miR141-3p expression was reduced in $\mathrm{CaOx}$ crystal-induced RTECs. To investigate the exact function of miR-141-3p in $\mathrm{CaOx}$ crystal-induced RTECs, we overexpressed miR141-3p in CaOx crystal-induced HK-2 cells. miR-141-3p overexpression notably reduced the apoptosis level, enhanced cell viability, decreased LDH and MDA contents, and increased SOD content. Briefly, miR-141-3p overexpression alleviated $\mathrm{CaOx}$ crystal-induced RTEC injury.

Pyroptosis is a form of proinflammatory cell death, leading to the release of cytokines to activate proinflammatory mediators [23]. Recent evidence has provided the mechanism underlying pyroptosis in $\mathrm{CaOx}$ crystal-induced renal calculi [24]. miR-141-3p can suppress high glucose-induced cardiomyocyte pyroptosis [15]. We speculated that miR-141-3p might alleviate CaOx crystalinduced RTEC injury by suppressing pyroptosis of RTECs. Pyroptosis is a functional result of NLRP3 inflammasome activation, which is mediated by the membrane pore-forming activity of GSDMD N-terminal domain secreted by caspase- 1 cleavage, thereby releasing massive proinflammatory cytokines such as IL- $1 \beta$ and IL18 [23]. Hence, we detected the pyroptosis-related factors in $\mathrm{CaOx}$ crystal-induced $\mathrm{HK}-2$ cells and found that $\mathrm{CaOx}$ crystals elevated the NLRP3 expression, enhanced the contents of IL-1 $\beta$ and IL-18, and increased the levels of cleaved caspase- 1 and GSDMD-N proteins in HK- 2 cells,

Fig. 5. NLRP3 overexpression reversed the protective effect of miR-141-3p overexpression on RTECs. RTEC injury was induced by $\mathrm{CaOx}$ crystals with a concentration of $2 \mathrm{~mm}$. CaOx crystal-induced RTECs were transfected with oe-NLRP3 and then co-treated with miR-141-3p overexpression for a combined experiment. a NLRP3 expression was determined using RT-qPCR and Western blot. b Cell viability was evaluated using the CCK-8 assay. c Apoptosis level was measured using flow cytometry. $\mathbf{d}$ The contents of LDH, MDA, and SOD were detected using ELISA. e The protein levels of NLRP3, cleaved caspase-1, and GSDMD-N were detected using Western blot. $\mathbf{f}$ The levels of IL- $1 \beta$ and IL- 18 were detected using ELISA. The cell experiment was repeated 3 times independently. Data are presented as mean \pm standard deviation. Data in panels $(\mathbf{a}-\mathbf{d})$ were analyzed using one-way ANOVA, and data in panels $(\mathbf{e}, \mathbf{f})$ were analyzed using two-way ANOVA, followed by Tukey's multiple comparisons test, ${ }^{* *} p<0.01$. RT-qPCR, reverse transcription quantitative polymerase chain reaction; ELISA, enzyme-linked immunosorbent assay; CCK-8, Cell Counting Kit-8.

miR-141-3p Reduces CaOx-Induced RTEC Injury while miR-141-3p overexpression notably reversed the above trends, suggesting that miR-141-3p overexpression suppressed $\mathrm{CaOx}$ crystal-induced pyroptosis of RTECs.

Thereafter, we focused on the downstream mechanism of miR-141-3p in CaOx crystal-induced RTEC injury. miR141-3p was predicted to have a binding site with NLRP3, and the dual-luciferase assay validated the binding of miR141-3p and NLRP3. CaOx crystals trigger renal inflammation via NLRP3-mediated IL-1 $\beta$ secretion [25]. NLRP3-deficient mice suffering from chronic oxalate nephropathy have reduced progressive renal failure and mortality [26]. Our results exhibited that $\mathrm{CaOx}$ crystals elevated NLRP3 expression, while miR-141-3p overexpression reduced NLRP3 expression, suggesting that miR-141-3p targeted NLRP3 in CaOx crystal-induced RTECs. Then, we overexpressed NLRP3 in HK-2 cells to verify that NLRP3 was involved in the protective effect of miR-141-3p on CaOx crystal-induced RTECs. NLRP3 inhibition can suppress the secretion of inflammatory mediators, thereby mitigating $\mathrm{CaOx}$ crystal-induced renal injury and crystal deposits in HK-2 cells [20]. Consistently, our results demonstrated that NLRP3 overexpression notably enhanced the apoptosis level, reduced cell viability, and increased the pyroptosis of RTECs. Briefly, NLRP3 overexpression reversed the protective effect of miR-141-3p overexpression on RTECs.

\section{Conclusion}

To sum up, miR-141-3p represses NLRP3 expression by directly binding to NLRP3, thereby suppressing NLRP3-mediated pyroptosis of RTECs. This finding may provide a theoretical basis for the clinical treatment of hyperoxaluria or idiopathic kidney stones. However, we merely verified this mechanism in cell experiments and failed to validate it in animal experiments. To explore the clinical significance of this mechanism in the diagnosis of hyperoxaluria or idiopathic kidney stones, we should further verify this mechanism in animal models (such as rats/mice). There are great differences between animal models (rats/mice) and humans in physiological structure, so more preclinical and clinical studies are needed. Hence, we will further verify the role of the miR-141-3p/ NLRP3 axis in hyperoxaluria or idiopathic kidney stones in animal models in future experiments and conduct clinical experiments according to the research results to explore the application value of this mechanism in hyperoxaluria or idiopathic kidney stones. Moreover, there still lacked in-depth study on the upstream mechanism of miR-141-3p and the downstream mechanism of NLRP3. 
In the future, we will also further explore the upstream genes of miR-141-3p and the downstream genes of NLRP3 in $\mathrm{CaOx}$ crystal-induced RTEC injury.

\section{Statement of Ethics}

The study does not involve the use of animals or human subjects.

\section{Conflict of Interest Statement}

The authors have no conflicts of interest to declare.

\section{Funding Sources}

Not applicable.

\section{References}

1 Khan SR, Pearle MS, Robertson WG, Gambaro G, Canales BK, Doizi S, et al. Kidney stones. Nat Rev Dis Primers. 2016 Feb 25;2:16008.

2 Zisman AL. Effectiveness of treatment modalities on kidney stone recurrence. Clin J Am Soc Nephrol. 2017 Oct 6;12(10):1699-708.

3 Gao H, Zhang H, Wang Y, Li K, Du W, Wang X, et al. Treatment of complex renal calculi by digital flexible ureterorenoscopy combined with single-tract super-mini percutaneous nephrolithotomy in prone position: a retrospective cohort study. Med Sci Monit. 2019 Aug 7;25:5878-85.

4 Zhu W, Liu Y, Lan Y, Li X, Luo L, Duan X, et al. Dietary vinegar prevents kidney stone recurrence via epigenetic regulations. EBioMedicine. 2019 Jul;45:231-50.

5 Huang Y, Zhang YH, Chi ZP, Huang R, Huang $\mathrm{H}$, Liu G, et al. The handling of oxalate in the body and the origin of oxalate in calcium oxalate stones. Urol Int. 2020;104(3-4):167-76.

6 Wang Z, Li MX, Xu CZ, Zhang Y, Deng Q, Sun $\mathrm{R}$, et al. Comprehensive study of altered proteomic landscape in proximal renal tubular epithelial cells in response to calcium oxalate monohydrate crystals. BMC Urol. 2020 Aug 31;20(1): 136.

7 Qi S, Wang Q, Xie B, Chen Y, Zhang Z, Xu Y. P38 MAPK signaling pathway mediates COM crystal-induced crystal adhesion change in rat renal tubular epithelial cells. Urolithiasis. 2020 Feb;48(1):9-18.

8 Narula S, Tandon S, Singh SK, Tandon C. Kidney stone matrix proteins ameliorate calcium oxalate monohydrate induced apoptotic injury to renal epithelial cells. Life Sci. 2016 Nov 1;164 23-30.

9 Mulay SR, Kulkarni OP, Rupanagudi KV, Migliorini A, Darisipudi MN, Vilaysane A, et al. Calcium oxalate crystals induce renal inflammation by NLRP3-mediated IL-1beta secretion. J Clin Invest. 2013 Jan;123(1):236-46.

\section{Author Contributions}

Xiu-Guo Gan has given substantial contributions to the conception and the design of the manuscript and Zhi-Hao Wang and Hai-Tao Xu contributed to acquisition, analysis, and interpretation of the data. All authors have participated to drafting the manuscript and Xiu-Guo Gan revised it critically. All authors read and approved the final version of the manuscript.

\section{Data Availability Statement}

All data generated or analyzed during this study are included in this article. Further inquiries can be directed to the corresponding author.
$10 \mathrm{HeY}$, Hara H, Núñez G. Mechanism and regulation of NLRP3 inflammasome activation. Trends Biochem Sci. 2016 Dec;41(12):1012-21.

11 Kim YG, Kim SM, Kim KP, Lee SH, Moon JY. The role of inflammasome-dependent and inflammasome-independent NLRP3 in the kidney. Cells. 2019 Nov 5;8(11):1389.

12 Lan C, Chen D, Liang X, Huang J, Zeng T, Duan $\mathrm{X}$, et al. Integrative analysis of miRNA and mRNA expression profiles in calcium oxalate nephrolithiasis rat model. Biomed Res Int. 2017; 2017:8306736.

13 Wang B, Wu B, Liu J, Yao W, Xia D, Li L, et al. Analysis of altered microRNA expression profiles in proximal renal tubular cells in response to calcium oxalate monohydrate crystal adhesion: implications for kidney stone disease. PLoS One. 2014;9(7):e101306

14 Zhang B, Zhao C, Hou L, Wu Y. Silencing of the IncRNA TUG1 attenuates epithelial-mesenchymal transition of renal tubular epithelial cells by sponging miR-141-3p via regulating $\beta$-catenin. Am J Physiol Renal Physiol. 2020 Dec 1;319(6): F1125-F34.

15 Wu A, Sun W, Mou F. IncRNAMALAT1 promotes high glucoseinduced $\mathrm{H} 9 \mathrm{C} 2$ cardiomyocyte pyroptosis by downregulating miR1413p expression. Mol Med Rep. 2021 Apr;23(4):259.

16 Wang XF, Zhang BH, Lu XQ, Wang RQ. Gastrin-releasing peptide receptor gene silencing inhibits the development of the epithelial-mesenchymal transition and formation of a calcium oxalate crystal in renal tubular epithelial cells in mice with kidney stones via the PI3K/Akt signaling pathway. J Cell Physiol. 2019 Feb;234(2): 1567-77.

17 Xu C, Zhang W, Lu P, Chen JC, Zhou YQ, Shen G, et al. Mutation of Klotho rs3752472 protect the kidney from the renal epithelial cell injury caused by $\mathrm{CaOx}$ crystals through the Wnt/betacatenin signaling pathway. Urolithiasis. 2021 May 29.
18 Agarwal V, Bell GW, Nam JW, Bartel DP. Predicting effective microRNA target sites in mammalian mRNAs. Elife. 2015 Aug 12;4:4.

19 Prummer JK, Howard J, Grandt LM, Obrador de Aguilar R, Meneses F, Peters LM. Hyperlipasemia in critically ill dogs with and without acute pancreatitis: Prevalence, underlying diseases, predictors, and outcome. J Vet Intern Med. 2020 Nov;34(6):2319-29.

20 Sun Y, Liu Y, Guan X, Kang J, Wang X, Liu Q, et al. Atorvastatin inhibits renal inflammatory response induced by calcium oxalate crystals via inhibiting the activation of TLR4/NF-kappaB and NLRP3 inflammasome. IUBMB Life. 2020 May;72(5):1065-74.

21 Alelign T, Petros B. Kidney stone disease: an update on current concepts. Adv Urol. 2018;2018: 3068365.

22 Khan SR. Reactive oxygen species as the molecular modulators of calcium oxalate kidney stone formation: evidence from clinical and experimental investigations. J Urol. 2013 Mar;189(3): $803-11$.

23 Liu X, Zhang Z, Ruan J, Pan Y, Magupalli VG, $\mathrm{Wu} \mathrm{H}$, et al. Inflammasome-activated gasdermin $\mathrm{D}$ causes pyroptosis by forming membrane pores. Nature. 2016 Jul 7;535(7610):153-8.

24 Song Z, Zhang Y, Gong B, Xu H, Hao Z, Liang C. Long noncoding RNA LINC00339 promotes renal tubular epithelial pyroptosis by regulating the miR-22-3p/NLRP3 axis in calcium oxalateinduced kidney stone. J Cell Biochem. 2019 Jun; 120(6): 10452-62.

25 Darisipudi MN, Knauf F. An update on the role of the inflammasomes in the pathogenesis of kidney diseases. Pediatr Nephrol. 2016 Apr; 31(4):535-44

26 Knauf F, Asplin JR, Granja I, Schmidt IM, Moeckel GW, David RJ, et al. NALP3-mediated inflammation is a principal cause of progressive renal failure in oxalate nephropathy. Kidney Int. 2013 Nov;84(5):895-901. 